\title{
TWO-STEP ITERATIVE METHOD FOR RADIATIVE TRANSFER CALCULATIONS IN AXISYMMETRIC FLAMES CONTAINING ABSORBING AND SCATTERING PARTICLES
}

\author{
Leonid A. Dombrovsky ${ }^{1,2^{*}}$, Siaka Dembele ${ }^{1}$, Jennifer X. Wen ${ }^{3}$, Ivan Sikic $^{3}$ \\ ${ }^{1}$ Department of Mechanical and Automotive Engineering, Kingston University, London, SW15 3DW, UK \\ ${ }^{2}$ Joint Institute for High Temperatures, 17A Krasnokazarmennaya St, NCHMT, Moscow, 111116, Russia \\ ${ }^{3}$ School of Engineering, University of Warwick, Coventry CV4 7AL, UK
}

\begin{abstract}
The discrete ordinates and finite volume methods used in most fire simulations codes for radiative transfer calculations suffer from high computing overheads. A procedure based on two-step iterative method is suggested to simplify time-consuming spectral radiative transfer calculations in open flames containing scattering particles. At the first step of the problem solution, the $P_{1}$ approximation is used to calculate the divergence of radiative flux, and it is sufficient to determine the flame parameters. The second step of solution is necessary to obtain the radiation field outside the flame, and this can be made independently using the raytracing procedure and the transport source function determined at the first step. Such a splitting of the complete problem results in much simpler algorithm than those used traditionally. It has been proved in previous papers that the combined two-step method is sufficiently accurate in diverse engineering applications, especially in the scattering media. At the same time, the computational time decreases in about two orders of magnitude as compared with direct methods. An axisymmetric pool fire suppressed by a water spray is considered as the case problem in the present paper to analyze the effect of radiation scattering by evaporating water droplets. The strong effect of scattering on radiative power field in the flame is obtained computationally for the first time.
\end{abstract}

KEY WORDS: Radiation, Computational methods, Large-scale open flames, Two-step method

\section{INTRODUCTION}

The radiative transfer calculations are the most time-consuming in computational fluid dynamics (CFD) simulation of fires. Therefore, the use of a simple but sufficiently accurate method for the radiative transfer is critically important to improve the computational costs of CFD codes. A numerical analysis of suppression of fires by water sprays is possible only with the use of spectral models because the optical properties of water droplets cannot be considered on the basis of a gray model. Fortunately, there is no need for a detailed radiation field in CFD calculations. Only the integral (over the spectrum) radiative flux divergence is important because it is a term in the energy equation. It means that one can consider a differential approach for radiative transfer instead of the radiative transfer equation (RTE). The first approximation of the method of spherical harmonics $\left(P_{1}\right)$ is the simplest method of this type, and there is a very positive long-time experience of using $P_{1}$ in similar multi-dimensional problems [1-5]. As a rule, the $P_{1}$ cannot give correct values of radiative flux near the open flame boundaries and the second step of the combined radiative transfer model is necessary. This can be done using the transport approximation [4] and a ray-tracing procedure for the RTE with known source function. The present study is focused on applicability of the earlier developed combined two-step method to the flames typical of pool fires under their suppression by water sprays, when the radiation scattering by evaporating droplets is important. A numerical solution for the conventional axisymmetric flame containing water droplets is considered because simplifications in the flame data are not important for the present study. 


\section{COMPUTATIONAL MODEL FOR THE CASE PROBLEM OF POOL FIRE}

One should take into account the effects of water sprays on the flow field parameters of the flame in the regular developed regime of fire suppression. However, it is not important in the first stage case of a specific procedure of fire suppression suggested by the authors in recent papers $[6,7]$. This procedure includes a preliminary probe stage with a very small flow rate of water. The observations at the probe stage could reveal some details of behavior of evaporating water droplets in the flame. Obviously, the effect of a water spray on the fire parameters at the short-time probe stage is negligible, and one can consider the motion, heating, and evaporation of single water droplets without taking into account any feedback effects.

The fire scenario considered in the present study is based on a $28 \mathrm{~cm}$ diameter methane flame above a pool of liquid fuel, burning in the open quiescent environment with a heat release rate of $53 \mathrm{~kW}$. The large eddy simulation (LES) was carried out using an in-house version of FireFOAM [8], compatible with OpenFOAM version 2.2.x. The combustion sub-model used in this code is based on the eddy dissipation concept (EDC) proposed by Magnussen et al. [9] for applications of the Reynolds averaged Navier-Stokes equations (RANS) extended by Chen et al. [10] for LES. For soot modelling, Chen [11] extended the laminar smoke point soot to LES using the partially stirred reactor concept. The radiative transfer equation is solved with the finite volume method (FVM), which is open accessible. Gas radiation is treated with the box model approach based on the exponential wide band model to calculate the equivalent band absorption coefficient $[12,13]$. Note that the most accurate numerical results could be obtained using the Monte Carlo simulation as it was done in [14] with the computer code developed by Surzhikov [15], but this method is even more time-consuming for regular spectral calculations at the moment.

The problem of fire suppression by water sprays is considered here. Therefore, the motion, heating, and evaporation of water droplets are also briefly considered in the paper. The radiative power absorbed by droplets is neglected because it is much less than the heat supplied by convective heat transfer to the droplets from hot combustion gases in the flame. The effect of radiation could be also taken into account in more detailed calculations as it was done in recent papers on shielding of fire radiation by water mists $[16,17]$ and also in the problem of a space vehicle shielding from solar radiation by a cloud of sublimating particles [18].

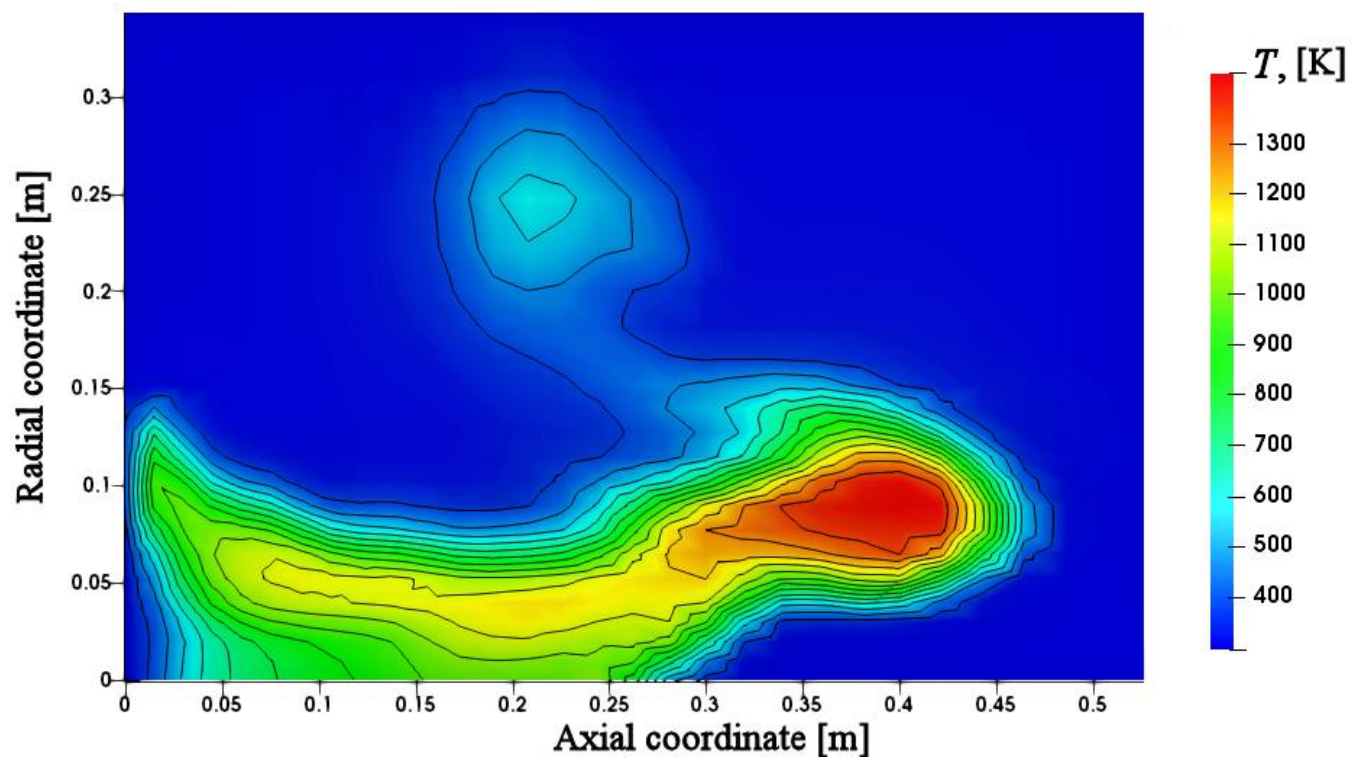

Fig. 1 The gas temperature field in the model flame.

The calculated transient flame is three-dimensional, but deviations of the main parameters from the axisymmetric case are not significant. Therefore, we consider similar axisymmetric fields obtained by an averaging in a selected axial cross section of the real developing flame at $t=1 \mathrm{~s}$ (see Fig. 1). 
The non-uniform triangulation of the computational region with 1600 elements is shown in Fig. 2. The smaller areas of finite elements were made in the region of more optically dense medium. As usually, the radial coordinates of all the nodes were increased by the first radial interval of the mesh to avoid the formal difficulties in numerical calculations near the axis [3].

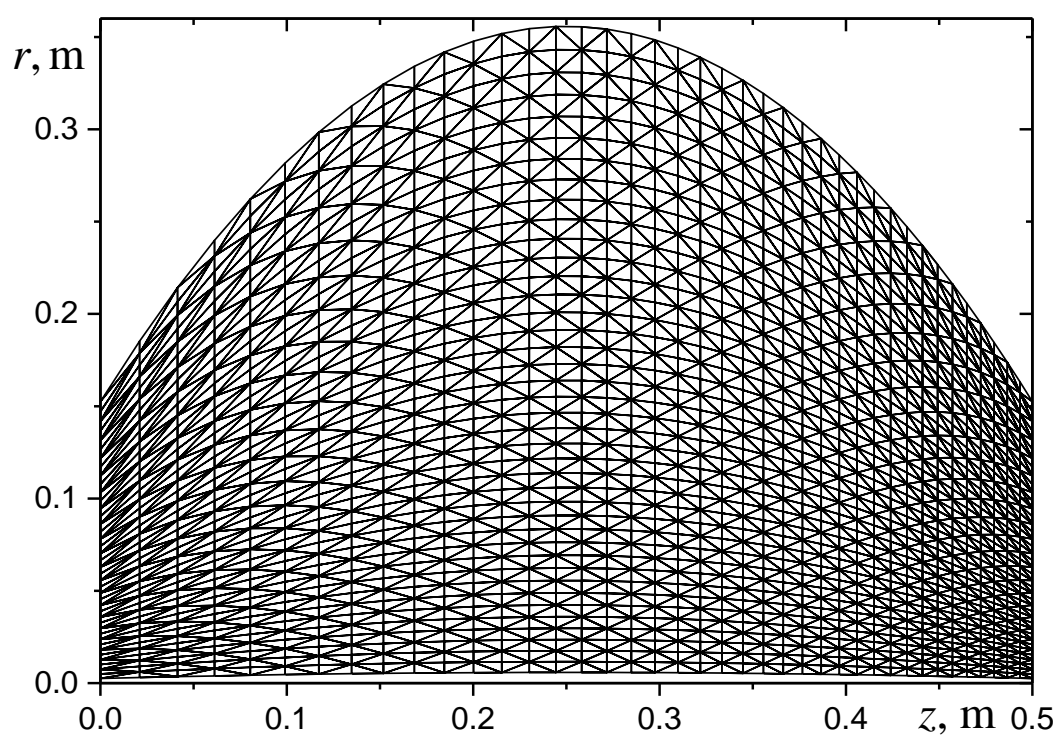

Fig. 2 Finite-element triangulation of the flame computational region.

\section{MOTION AND EVAPORATION OF WATER DROPLETS IN THE FLAME}

The interaction of water sprays with fires has been modeled computationally in many papers during the last two decades, but there is no need to discuss here the state-of-the-art in this field because the main focus is on the radiative transfer problem. Of course, there is a size distribution of water droplets at the initial cross section of the spray. However, it is convenient to obtain solutions for monodisperse droplets with sizes corresponding to conventional boundaries of size distribution. The trajectories of single droplets moving along the flame axis are considered. The initial problem for the droplet motion is as follows [19, 20]:

$$
\begin{gathered}
\frac{d z_{\mathrm{d}}}{d t}=u_{\mathrm{d}}, z_{\mathrm{d}}(0)=z_{0} \quad \frac{d u_{\mathrm{d}}}{d t}=\frac{3 C_{\mathrm{D}}}{8 a} \frac{\rho}{\rho_{\mathrm{w}}}\left(u-u_{\mathrm{d}}\right)\left|u-u_{\mathrm{d}}\right|-g, u_{\mathrm{d}}(0)=-u_{\mathrm{d} 0} \\
C_{\mathrm{D}}=24\left(1+0.15 \operatorname{Re}_{\mathrm{d}}^{0.687}\right) / \operatorname{Re}_{\mathrm{d}} \quad \operatorname{Re}_{\mathrm{d}}=2 \rho\left|u-u_{\mathrm{d}}\right| a / \eta
\end{gathered}
$$

It is assumed that water droplets are first heated up to the saturation temperature (at $0<t<t_{\text {sat }}$ ):

$$
\frac{d T_{\mathrm{d}}}{d t}=\frac{1.5 \mathrm{Nu} k}{\rho_{\mathrm{w}} c_{\mathrm{w}} a^{2}}\left(T-T_{\mathrm{d}}\right), T_{\mathrm{d}}(0)=T_{0} \quad \mathrm{Nu}=2+0.6 \operatorname{Re}_{\mathrm{d}}^{1 / 2} \operatorname{Pr}^{1 / 3} \quad \operatorname{Pr}=\eta c / k
$$

and then evaporated according to the following equation (at $t>t_{\text {sat }}$ ):

$$
\frac{d a}{d t}=-\mathrm{Nu} \frac{k\left(T-T_{\mathrm{sat}}\right)}{2 a \rho_{\mathrm{w}} L_{\mathrm{w}}} \quad a\left(t_{\mathrm{sat}}\right)=a_{0}
$$

This is a realistic assumption because of a large molar fraction of water vapor in the flame. A transfer from the droplet heating to its evaporation is given by equation $T_{\mathrm{d}}\left(t_{\text {sat }}\right)=T_{\text {sat }}$. The local relative volume fraction of water droplets can be calculated as:

$$
\bar{f}_{\mathrm{v}}=f_{\mathrm{v}} / f_{\mathrm{v} 0}=\left(u_{\mathrm{d} 0} / u_{\mathrm{d}}\right)\left(a / a_{0}\right)^{3}
$$

The examples of such calculations for the case problem can be found in [6,7]. The behavior of water droplets moving along the parallel lines appeared to be quite different, but in all cases the evaporating droplets of 
different initial sizes are focused on small local regions. In the case of droplets moving along the axis, their initial velocity makes no difference because of a relatively small relaxation time, and the final evaporation of these droplets is expected due to absorption of thermal radiation from a relatively hot region shown in Fig. 1. On the contrary, the large droplets supplied at $r_{0}=0.1 \mathrm{~m}$ can penetrate to the hot region and totally evaporate during their backward motion at a small distance from this region [7]. It is physically clear that the volume fraction of water droplets increases dramatically in the local regions of their collections and total evaporation. Strictly speaking, the assumption of independent motion of single droplets may be incorrect in the vicinity of these regions. Following [6, 7], this effect is not considered in the present paper.

\section{OPTICAL PROPERIES OF WATER DROPLETS}

The normalized coefficients of absorption and transport scattering (per unit volume fraction of water) for independently absorbing and scattering droplets with radius $a$ can be determined as follows:

$$
E_{\mathrm{a}}=0.75 Q_{\mathrm{a}} / a \quad E_{\mathrm{s}}^{\mathrm{tr}}=0.75 Q_{\mathrm{s}}^{\mathrm{tr}} / a
$$

According to Mie theory [21], the efficiency factors of absorption, $Q_{\mathrm{a}}$, and transport scattering, $Q_{\mathrm{s}}^{\mathrm{tr}}$ depend on both the complex index of refraction of water, $m=n-i \kappa$, and diffraction parameter $x=2 \pi a / \lambda$. Following $[16,17]$ the approximate relations suggested in [22] are used in the calculations:

$$
\begin{gathered}
Q_{\mathrm{a}}=\frac{4 n}{(n+1)^{2}}[1-\exp (-4 \kappa x)] \quad Q_{\mathrm{s}}^{\mathrm{tr}}=\xi\left\{\begin{array}{c}
\varphi / 5 \text { when } \varphi \leq 5 \\
(5 / \varphi)^{\gamma}
\end{array} \quad \text { when } \varphi>5\right. \\
\xi=1.5 n(n-1) \exp (-15 \kappa) \quad \gamma=1.4-\exp (-80 \kappa) \quad \varphi=2 x(n-1)
\end{gathered}
$$

The calculated values of $E_{\mathrm{a}}$ and $E_{\mathrm{s}}$ presented in Fig. 3 should be multiplied by the volume fraction of droplets to obtain the local values of the spectral absorption and transport scattering coefficients: $\alpha_{\mathrm{d}}=f_{\mathrm{v}} E_{\mathrm{a}}$ and $\sigma_{\mathrm{tr}}=f_{\mathrm{v}} E_{\mathrm{s}}^{\mathrm{tr}}$. The radiation absorption by water droplets may be significant in the near-infrared absorption bands. It contributes to water evaporation and flame suppression. The scattering by small droplets is large over the spectrum excluding the absorption band, where the anomalous dispersion takes place. The hypothesis of independent scattering does not work in the region of strong evaporation where the distance between the neighboring droplets may be comparable with the wavelength. The dependent scattering may lead to significant changes in optical properties of the medium [23, 24]. However, this effect is a complex problem of physical optics and it is beyond the scope of the present paper. Therefore, the ordinary relations of independent scattering by single droplets are used in the estimates.
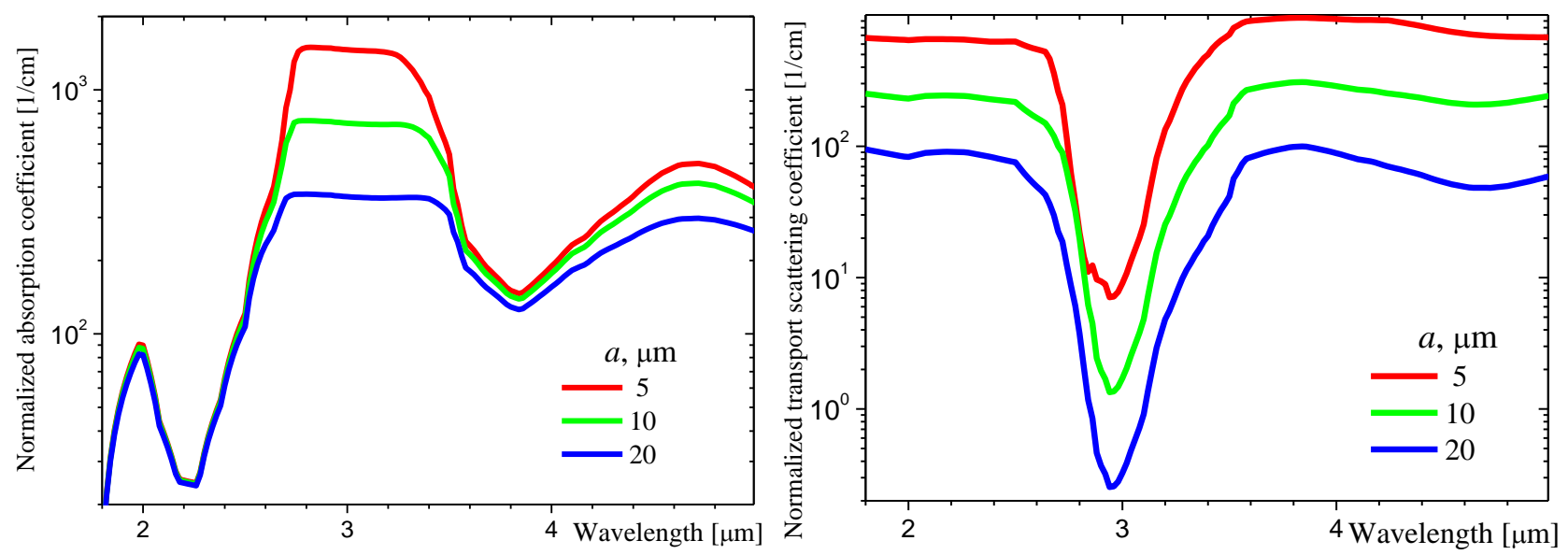

Fig. 3 Absorption and transport scattering coefficients of water droplets. 


\section{TWO-STEP METHOD FOR RADIATIVE TRANSFER CALCULATIONS}

The $P_{1}$ method for radiative transfer can be employed in the CFD flame calculations, whereas a ray-tracing procedure for the transport RTE solution can be used at the second step to calculate the radiative flux outside the flame. Such an approach will simplify significantly the calculations. Note that the finite-element method (FEM) [25] can be used to solve the $P_{1}$ problem [1, 3]. The FEM is very flexible and applicable for computational regions of complex shape. Mathematically, the $P_{1}$ leads to the nonhomogeneous modified Helmholtz equation for the spectral irradiation [3] (the subscript $\lambda$ is hereafter omitted for brevity):

$$
-\nabla(D \nabla G)+\alpha G=4 \alpha F \quad D=1 /\left(3 \beta_{\mathrm{tr}}\right) \quad F(T)=\pi \mathrm{I}_{\mathrm{b}}(T)
$$

where $D, \beta_{\mathrm{tr}}=\alpha+\sigma_{\mathrm{tr}}$ are the coefficients of radiation diffusion and transport extinction, $I_{\mathrm{b}}(T)$ is the Planck function. The Marshak conditions are used to complete the problem. One can proceed to the variational formulation of the problem when the solution yields the minimum of the functional [3]:

$$
\chi=\int_{V}\left[\frac{D}{2} \nabla G+\frac{\alpha}{2} G^{2}-4 \alpha F(T) G\right] d V+\int_{S} \frac{\varepsilon_{\mathrm{w}}}{2\left(2-\varepsilon_{\mathrm{w}}\right)}\left[G^{2} / 2-4 F\left(T_{\mathrm{w}}\right) G\right] d S
$$

where $T_{\mathrm{w}}$ and $\varepsilon_{\mathrm{w}}$ are respectively the temperature and hemispherical emissivity of boundary surfaces. The order of the differential operator in Eq. (7) is reduced by one compared with Eq. (8). As a result, the local linear approximations of function $G$ in triangle finite elements are used. The transport RTE

$$
\vec{\Omega} \nabla I(\vec{r}, \vec{\Omega})+\beta_{\mathrm{tr}} I(\vec{r}, \vec{\Omega})=\frac{\sigma_{\mathrm{tr}}}{4 \pi} G(\vec{r})+\alpha I_{\mathrm{b}}(T) \quad G(\vec{r})=\int_{(4 \pi)} I(\vec{r}, \vec{\Omega}) d \vec{\Omega}
$$

is then solved using a ray-tracing procedure to obtain the radiative flux from the flame.

\section{EFFECT OF RADIATION SCATTERING BY EVAPORATING WATER DROPLETS}

There is no need in complete calculations to estimate the effect of scattering. It is sufficient to consider spectral results at the wavelength of $\lambda=4.2 \mu \mathrm{m}$, near the peak of the $\mathrm{CO}_{2}$ absorption band. These spectral calculations are representative because of significant contribution of the considered spectral range to the integral flame radiation and the expected valuable contribution of radiation scattering by water droplets. The radiation absorption by droplets is very small in this range, whereas the normalized transport scattering coefficient $E_{\mathrm{s}}^{\mathrm{tr}} \approx 300 \mathrm{~cm}^{-1}$ at $a=10 \mu \mathrm{m}$ and $1000 \mathrm{~cm}^{-1}$ at $a=5 \mu \mathrm{m}$ (see Fig. 3). The estimates at realistic value of $f_{\mathrm{v}}=5 \cdot 10^{-4}$ give $\sigma_{\mathrm{tr}}=0.15 \mathrm{~cm}^{-1}$ in the region of $r<5 \mathrm{~cm}, 30<z<50 \mathrm{~cm}$ and $\sigma_{\mathrm{tr}}=0.5 \mathrm{~cm}^{-1}$ in the region of

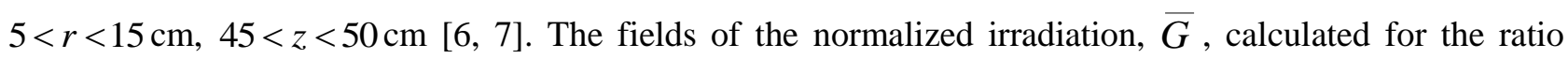
$F / F\left(T_{*}\right)$ where $T_{*}=1000 \mathrm{~K}$ are presented in Fig. 4. According to [14], the emissivity of the fuel pool surface was taken equal to 0.6 . The boundary of $r[\mathrm{~m}]=0.35-3.2(z[\mathrm{~m}]-0.25)^{2}$ and a non-uniform triangulation with 1600 finite elements was used in FEM calculations [7]. The calculations were performed on laptop with processor Intel Core i5-6300U@2.40GHz and the time of one calculation appeared to be about one second or even less. This is a very important advantage of the present method for multiple calculations of the radiative flux divergence in CFD modeling of pool fires as compared with the much more time-consuming calculations using the finite volume method employed in the CFD code FireFOAM. A comparison of the $P_{1}$ and the reference FVM computational results is beyond the scope of the present paper and will be a subject of a subsequent separate study. As to the calculations of the radiative flux from the flame, this is the second more complicated step of the suggested iterative method. However, these calculations can be done separately, after the CFD numerical solution.

One can see in Fig. 4 that a dense water mist leads to the strong changes in the irradiation field and increases significantly the maximum irradiation value in the flame. To the best of our knowledge, this effect, which may be used in optical diagnostics of transient flames at the probe stage of fire suppression, was obtained for the first time. 

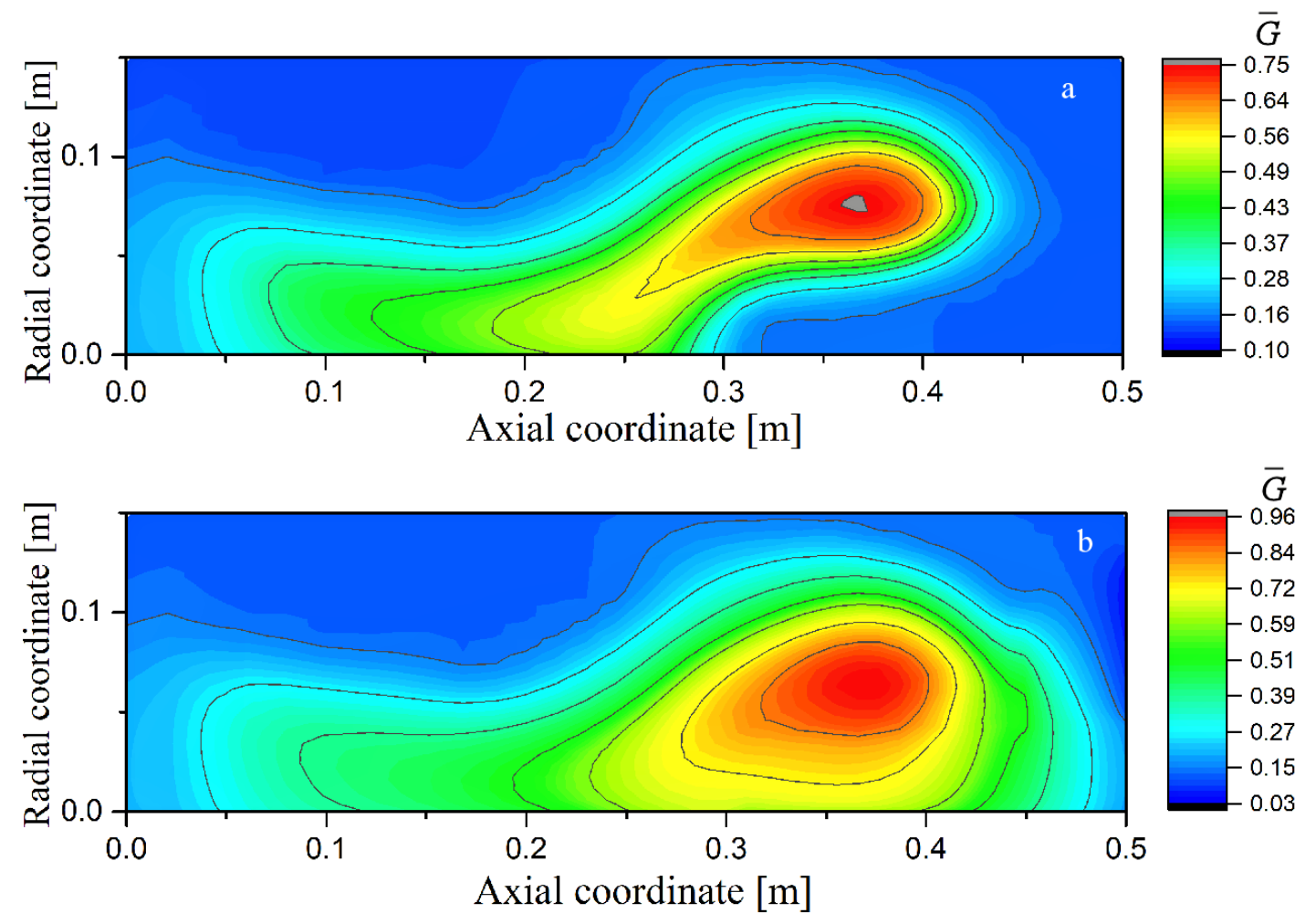

Fig. 4 The normalized spectral irradiation: $a$ - without water droplets, $b$ - with water droplets.

\section{CONCLUSIONS}

A procedure based on the earlier developed combined two-step method is suggested to simplify timeconsuming spectral radiative transfer calculations in transient pool fires during their suppression using water sprays. This can be considered as an important step in improving fire radiation modeling. The calculations for real developing flame showed for the first time that the effect of radiation scattering by evaporating water droplets on the radiation field in the flame may be significant. This finding should be taken into account in further studies of suppression of open flames by water sprays.

\section{ACKNOWLEDGMENTS}

The authors gratefully acknowledge the financial support by the H2020-MSCA-IF-2016 Programme of the European Commission (RAD-FIRE project, no. 749220). We also wish to thank Antoine Hubert for his kind assistance in processing the pool fire data.

\section{NOMENCLATURE}

$\begin{array}{llc}a & \text { radius of water droplet } & (\mu \mathrm{m}) \\ c & \text { specific heat capacity } & (\mathrm{J} / \mathrm{kg} / \mathrm{K}) \\ D & \text { radiation diffusion coefficient } & \left(\mathrm{m}^{-1}\right) \\ E & \text { normalized coefficient } & \left(\mathrm{m}^{-1}\right) \\ F & \text { function introduced by Eq. }(7) & \left(\mathrm{W} / \mathrm{m}^{2} / \mu \mathrm{m} / \mathrm{ster}\right) \\ f_{\mathrm{v}} & \text { volume fraction of droplets } & (-) \\ G & \text { spectral irradiation } & \left(\mathrm{W} / \mathrm{m}^{2} / \mu \mathrm{m}\right) \\ g & \text { acceleration of gravity } & \left(\mathrm{m} / \mathrm{s}^{2}\right)\end{array}$

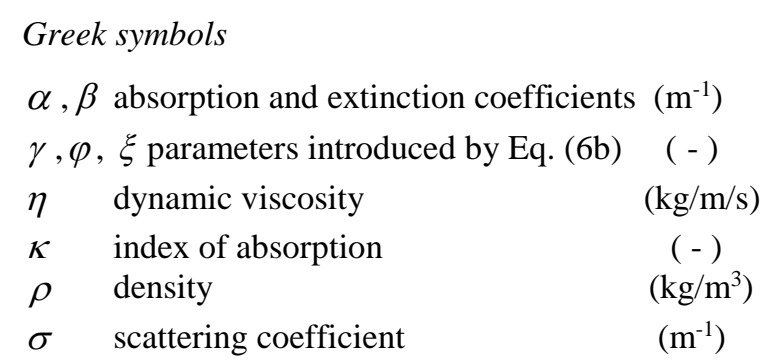




$\begin{array}{llclll}I & \text { spectral radiation intensity } & \left(\mathrm{W} / \mathrm{m}^{2} / \mu \mathrm{m} / \mathrm{ster}\right) & \chi & \text { functional introduced by Eq. }(8) & (\mathrm{W} / \mathrm{m} / \mu \mathrm{m}) \\ k & \text { thermal conductivity } & (\mathrm{W} / \mathrm{m} / \mathrm{K}) & & \\ m=n-i \kappa \quad \text { complex index of refraction } & (-) & \text { Subscripts and superscripts } \\ n & \text { index of refraction } & (-) & \mathrm{a} & \text { absorption } \\ Q & \text { efficiency factor } & (-) & \mathrm{b} & \text { blackbody } \\ r & \text { radial coordinate } & (\mathrm{m}) & \mathrm{d} & \text { droplet } \\ u & \text { velocity } & (\mathrm{m} / \mathrm{s}) & \mathrm{s} & \text { scattering } \\ x & \text { diffraction parameter } & (-) & \mathrm{tr} & \text { transport } \\ z & \text { axial coordinate } & (\mathrm{m}) & \mathrm{w} & \text { wall }\end{array}$

\section{REFERENCES}

[1] Dombrovsky, L.A., "Approximate methods for calculating radiation heat transfer in dispersed systems," Thermal Engineering 43(3), pp. 235-243, (1996).

[2] Dombrovsky, L.A. and Lipiński, W., "A combined $P_{1}$ and Monte Carlo model for multi-dimensional radiative transfer problems in scattering media," Comput. Thermal Sci. 2 (6), pp. 549-560, (2010).

[3] Dombrovsky, L.A. and Baillis, D., Thermal Radiation in Disperse Systems: An Engineering Approach, New York: Begell House, (2010).

[4] Dombrovsky, L.A., "The use of transport approximation and diffusion-based models in radiative transfer calculations," Comput. Therm. Sci. 4(4) pp. 297-315, (2012).

[5] Dombrovsky, L.A., Reviznikov, D.L., and Sposobin, A.V., "Radiative heat transfer from supersonic flow with suspended particles to a blunt body," Int. J. Heat Mass Transf. 93, pp. 853-861, (2016).

[6] Dombrovsky, L.A., Dembele, S., Wen J.X., and Sikic I., "Suppression of pool fires by water sprays: The effect of light scattering by evaporating droplets," Proc. $6^{\text {th }}$ Int. Conf. Comput. Thermal Radiation in Participating Media (CTRPM-VI), April 9-11, Cascais, Portugal, paper 30, (2018).

[7] Dombrovsky, L.A., Dembele, S., and Wen J.X., "An infrared scattering by evaporating droplets at the initial stage of a pool fire suppression by water sprays,” Infrared Phys. Technol., 2018 (in press).

[8] Sikic, I., Wen, J.X., and Dembele, S., "Evaluation of different schemes of the weighted-sum-of-grey-gases model for fire simulations," Proc. $9^{\text {th }}$ US Nat. Combust. Meeting, Combust. Inst., (2015).

[9] Magnussen, B.F. and Hjertager, B.H., "Development of the eddy-break-up model of turbulent combustion," Proc. Combust. Inst., 16, pp. 719-729, (1976).

[10] Chen, Z., Wen, J., Xu, B., and Dembele, S., "Large eddy simulation of a medium-scale methanol pool fire using the extended eddy dissipation concept," Int. J. Heat Mass Transf., 70, pp. 389-408, (2014).

[11] Chen, Z.B., "Extension of the eddy dissipation concept and laminar smoke point soot model to the large eddy simulation of fire dynamics," PhD thesis, Kingston University London, UK, (2012).

[12] Edwards, D.K., "Molecular gas radiation," in "Advances in Heat Transfer", 12, pp. 115-193, New York: Acad. Press, (1976).

[13] Modest, M.F., Radiative Heat Transfer, Third edition, New York: Acad. Press, (2013).

[14] Snegirev, A.Yu., "Statistical modelling of thermal radiation transfer in buoyant turbulent diffusion flames," Combust. Flame 136 (1-2), pp. 51-71, (2004).

[15] Surzhikov, S.T. and Howell, J.R., "Monte Carlo simulation of radiation in scattering volumes with line structure," AIAA J. Thermophys. Heat Transf. 12(2), pp. 278-281, (1998).

[16] Dombrovsky, L.A., Dembele, S., and Wen, J.X., "A simplified model for the shielding of fire thermal radiation by water mists," Int. J. Heat Mass Transf. 96, pp. 199-209, (2016).

[17] Dombrovsky, L.A., Dembele, S., Wen, J.X., "Shielding of fire radiation with the use of multi-layered mist curtains: preliminary estimates," Comput. Thermal Sci. 8(4) pp. 371-380, (2016).

[18] Dombrovsky, L.A., Reviznikov, D.L., Kryukov, A.P., and Levashov V.Yu., "Self-generated clouds of micron-sized particles as a promising way of a solar probe shielding from intense thermal radiation of the Sun," J. Quant. Spectr. Radiat. Transf. 200, pp. 234-243, (2017).

[19] Hua, J., Kumar, K., Khoo, B.C., and Xue, H., “A numerical study of the interaction of water spray with fire plume," Fire Safety J. 37, pp. 631-657, (2002).

[20] Crowe, C.T., Schwarzkopf, J.D., Sommerfeld, M., and Tsuji, Y. Multiphase Flows with Droplets and Particles, Second edition, New York: CRC Press, (2011).

[21] Bohren, C.F. and Huffman, D.R., Absorption and Scattering of Light by Small Particles, New York: Wiley, (1983).

[22] Dombrovsky, L.A., "Spectral model of absorption and scattering of thermal radiation by diesel fuel droplets," High Temper. 40(2), pp. 242-248, (2002).

[23] Mishchenko, M.I., Travis, L.D., and Lacis A.A., Multiple Scattering of Light by Particles: Radiative Transfer and Coherent Backscattering, Cambridge Univ. Press, (2006).

[24] Okada, Y. and Kokhanovsky, A.A., "Light Scattering and Absorption by Densely Packed Groups of Spherical Particles," J. Quant. Spectr. Radiat. Transf. 110(11), pp. 902-917, (2009).

[25] Zienkiewicz, O.C., Taylor, R.L., and Zhu, J.Z., The Finite Element Method: Its Basis and Fundamentals, Seventh Edition, New York: Elsevier, (2015). 